\title{
Effect of synthetic jet parameters on flow control of an aerofoil at high Reynolds number
}

\author{
JIANJUN FENG*, YUAN LIN, GUOJUN ZHU and XINGQI LUO \\ State Key Laboratory of Eco-hydraulics in Northwest Arid Region of China, Xi'an University of Technology, \\ Xi' an 710048, China \\ e-mail: jianjunfeng@xaut.edu.cn
}

MS received 29 September 2018; revised 28 May 2019; accepted 21 June 2019

\begin{abstract}
In this work, flow separation control has been conducted for the S809 aerofoil at a high Reynolds number using synthetic jet technology. The aerodynamic characteristics of the aerofoil have been compared in detail at different angles of attack, for the cases with and without adoption of synthetic jet. Numerical methods are employed for predicting flow structure and performance of the aerofoil. In addition, main parameters of the synthetic jet are optimized by the orthogonal experimental design, and dual jets are also employed for the comparison to a single jet. The results show that the flow separation at large angles of attack can be eliminated or greatly reduced by the synthetic jet, due to the mixing of low-energy fluid in boundary layer with high-energy fluid produced by the synthetic jet. The lift-to-drag ratio has been considerably increased by the synthetic jet for the critical condition, deep stall condition and complete stall condition as well. The maximum jet velocity of the synthetic jet is found to have the biggest effect on flow separation control. Furthermore, compared with single synthetic jet, the dual jets can make much better improvement on flow separation control of the aerofoil, especially at the complete stall condition.
\end{abstract}

Keywords. Aerofoil; synthetic jet; flow control; numerical simulation; orthogonal experimental design.

\section{Introduction}

With the increase of attack angle, the lift-to-drag ratio of an aerofoil will increase gradually. When the angle of attack exceeds a critical value, a large inverse pressure gradient will be produced on the suction surface of the aerofoil. Consequently, flow separation will occur, which will reduce the lift and dramatically increase the drag of the aerofoil. Flow separation will not only cause energy loss to the flow but also produce noise and vibrations.

Flow control technology can effectively improve the flow pattern of an aerofoil, which can be divided into two categories: active control and passive control. Passive flow control does not require additional energy intervention and is simple to use, such as eddy current generator and grooves. However, the passive flow control cannot adapt to the variation of the inflow condition. Active flow control is a kind of controlling mode with auxiliary energy injection, in which appropriate disturbance is directly injected into the flow to interact with the main flow for achieving the purpose of flow control. Its control characteristics can be adjusted according to different flow situation. As a new type of active flow control technology,

*For correspondence

Published online: 27 July 2019 zero-mass jet has been paid more and more attention due to many distinct advantages such as small volume, light mass, no additional source injection and simple manufacturing. Many researchers have conducted lots of research work on zero-mass jet both experimentally $[1,2]$ and numerically [3, 4].

Synthetic jet (SJ) technology is also called zero-mass jet technology because it does not require extra energy injection. At present, this technology has been widely used in various engineering fields [5-9]. Smith and Glezer [10] developed successfully a synthetic jet actuator (SJA), which is schematically shown in figure 1 , and applied it to flow control. The jet actuator consists of a metal film, piezoelectric material, a cavity and a jet hole. Piezoelectric materials produce a periodic motion of the metal film and compress the volume of the cavity, and the periodic suction will be formed at the orifice of the jet. The gas will form a vortex pair during this continuous suction process, which will mix the low-velocity fluid in the bottom layer of the boundary layer with the high-energy fluid in the mainstream. The energy at the bottom of the boundary layer is strengthened, thus reducing the pressure gradient and delaying the flow separation phenomenon.

In recent years, with the development of micro-electromechanical technology, SJ technology has been 


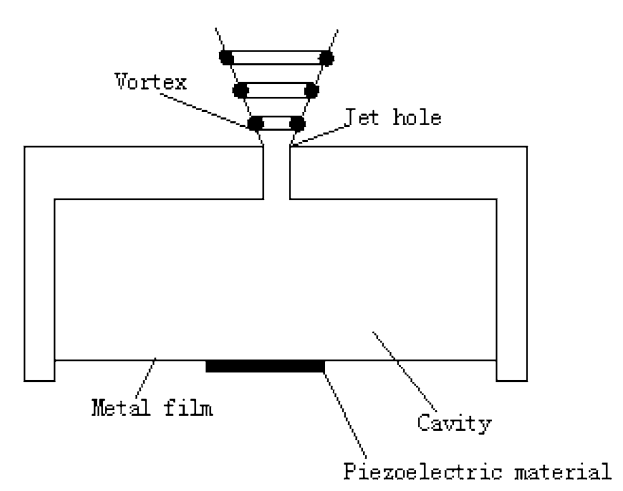

Figure 1. Synthetic jet actuator.

developed rapidly. Some work has been conducted to investigate the mechanism of jet [11-14]. Seifert et al [15] applied the jet actuator to an aerofoil, and the aerodynamic performance of the aerofoil was improved obviously. Qin et al [16] used end wall SJ to control the flow separation of stator cascade of high-load compressor. The results showed that the end wall SJ can significantly improve the flow in the blade channel. Under the design conditions, the maximum loss is reduced by 21.63 and the pressure increment is $5.6 \%$. Kamari and Tadjfar [17] performed flow control optimization for SD7003 aerofoil using an SJ tangential to boundary layer. The genetic algorithm was used to optimize jet parameters with the maximum performance as the objective function. Velasco et al [18] applied the method of active flow control to simulate the flow around a cross-flow vertical-axis turbine, demonstrating that the net torque and power output of the turbine can be improved using SJ outside the aerofoil and inside the aerofoil. Gilarranz and co-workers $[19,20]$ experimentally studied the flow separation and stall control of NACA0015 aerofoil at different angles of attack using a high-energy jet actuator. The results showed that the lift curve increased with increasing excitation frequency at small angle of attack. Under the excitation control of SJA, the stalling attack angle increased from $12^{\circ}$ to $18^{\circ}$. Moreover, Zhao et al [21] examined numerically the effects of jet control parameters on the dynamic stall characteristics of the OA212 rotor aerofoil.

All the mentioned work has contributed to the understanding and application of SJ on flow control. However, the comprehensive analysis and optimal design of main parameters affecting the control effect of the jet still need to be further investigated. In this paper, the aerodynamic characteristics of S809 aerofoil under flow separation controlling by SJs are examined in detail. The main parameters affecting jet effect, such as jet frequency, jet angle and maximum jet velocity, are comprehensively analysed. The parameters of the SJ are optimized by orthogonal experimental design (OED) method. Furthermore, the controlling effects between a single jet and dual ones are also compared and analysed.

\section{Numerical methodology}

\subsection{Computational model and grid generation}

The S809 aerofoil [22] is chosen for investigation. As shown in figure 2, the computing domain is C-typed. The distance between the computational domain boundary and the chord centre of the aerofoil is $10 c(c=0.6 \mathrm{~m}$ is the aerofoil chord length).

The grid generation tool ANSYS ICEM-CFD is applied to generate high-quality structured grids for the mentioned computational domain. As shown in figure 3, a combination of $\mathrm{H}$-grid and $\mathrm{C}$-grid is adopted for the grids. The $\mathrm{C}$-grid near the surface of the aerofoil can provide a better resolution on boundary layers.

For the boundary conditions, no-slip and hydraulically smooth conditions are specified on the aerofoil surface. For a hydraulically smooth condition, the thickness of viscous sublayer in the boundary layer is larger than the height of the wall roughness, and is therefore enough to cover the rough surface of the wall. As a result, the disturbance effect of the wall roughness on the flow can be almost neglected. The incoming flow velocity is $U_{\infty}=27.38 \mathrm{~m} / \mathrm{s}$ specified at the inlet, with Reynolds number $\operatorname{Re}=1 \times 10^{6}$ based on the chord length $c$. At the outlet, static pressure is given. For the turbulence condition, a turbulence intensity (ratio of the fluctuating part to the mean velocity) of $5 \%$ is set at the inlet, together with a viscosity ratio (ratio of the turbulence viscosity to dynamic viscosity) of 10 . In addition, an SJA that is shown in figure 1 is employed on the aerofoil for the flow control. The jet hole in a width of $1 \% \mathrm{c}$ is placed on the upper surface of the aerofoil, with a distance of $12 \% c$ to the aerofoil leading edge. The initial parameters of the SJ are selected based on Refs. [23, 24], and will be optimized later. The jet frequency is set to be $f=75 \mathrm{~Hz}$, and the angle between the jet flow and the tangential direction of the aerofoil upper surface is $\theta=30^{\circ}$. The periodic velocity $V_{j e t}$ is introduced at the jet hole according to Eq. (1), with a maximum value of $V_{\max }=2.25 U_{\infty}$. Furthermore, the lift and drag coefficients $C_{L}$ and $C_{D}$ are defined in Eqs. (2) and (3), respectively. The pressure coefficient $C_{P}$ is also specified in Eq. (4):

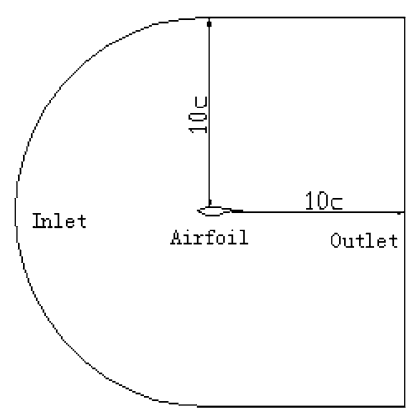

Figure 2. Computational model. 

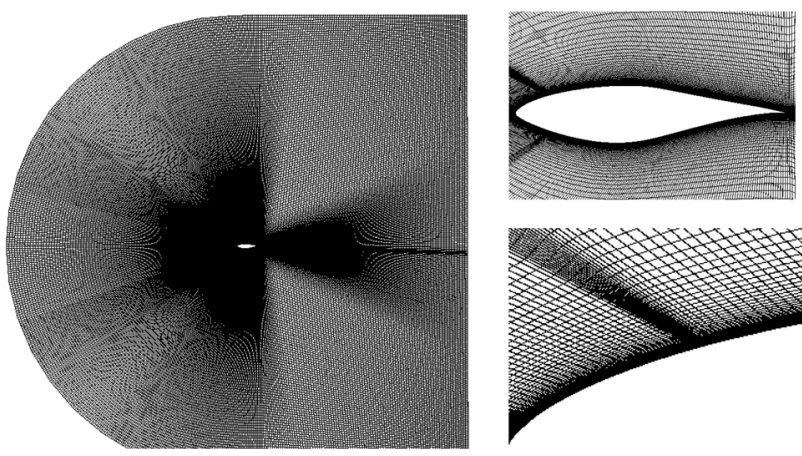

Figure 3. View of computational girds.

$$
\begin{gathered}
V_{j e t}(t)=V_{\max } \cdot \sin (2 \pi f t) \\
C_{L}=F_{L} /\left(0.5 \rho c U_{\infty}^{2}\right) \\
C_{D}=F_{D} /\left(0.5 \rho c U_{\infty}^{2}\right) \\
C_{P}=\left(P-P_{o}\right) /\left(0.5 \rho U_{\infty}^{2}\right)
\end{gathered}
$$

The CFD code ANSYS CFX-18 is used to solve the unsteady N-S equations. The SST turbulence model [25] is chosen to simulate the turbulent flow, which is a combination of $k-\omega$ model near wall boundary and $k-\varepsilon$ model for the main flow. This model is assumed to be able to accurately predict the onset and amount of flow separations under the condition of adverse pressure gradients by including the transport effects into the formulation of the eddy viscosity. The second-order backward Euler scheme is chosen for the time term, the second-order format is applied for the discretization in space and other terms.

\section{Results and discussion}

In this paper, all presented results are limited to the Reynolds number of $\mathrm{Re}=1 \times 10^{6}$ for the $\mathrm{S} 809$ aerofoil under different angles of attack.

Figure 4 shows the lift coefficient $\left(C_{L}\right)$ and drag coefficient $\left(C_{D}\right)$ with different mesh sizes for the condition near critical stall. It is found that when the mesh size reaches 100,000 , there is no evident difference either in the two mentioned coefficients or in flow structure, with the further increase in mesh size. Therefore, the total mesh size number of 100,000 is assumed to be sufficient for the current case, which can ensure a non-dimensional wall distance $y+$ below 10 in the whole computational domain, in order to obtain a better boundary resolution with the selected SST turbulence model.

In order to validate the applied numerical method, the predicted lift and drag coefficients are compared with experimental data provided by Somers [22], shown in figure 5, for the case without applying flow control. In

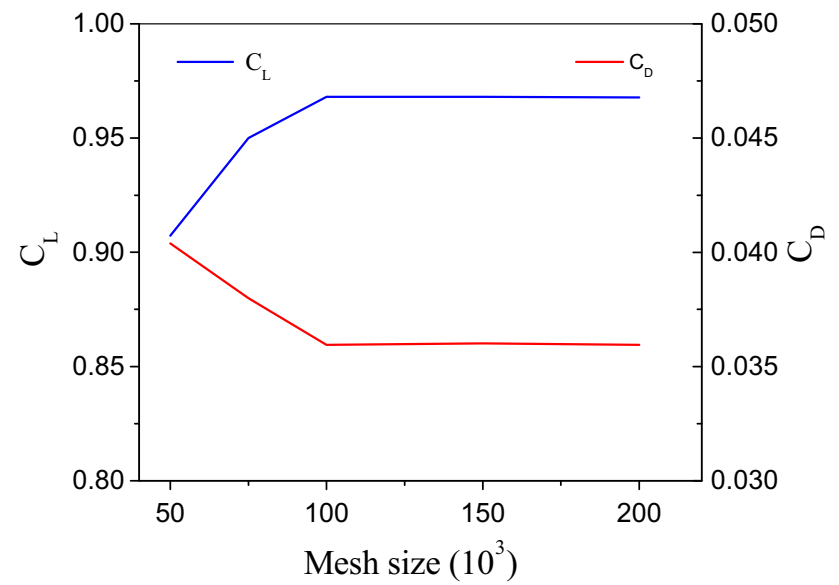

Figure 4. Grid independence study near critical stall condition.

addition, the CFD results are presented for both the SST and $k-\varepsilon$ models for comparison. It can be observed that the predicted lift and drag coefficients with the SST turbulence model agree generally well with experimental ones. Although some discrepancies are observed in the drag coefficient at high angle of attack between CFD and experimental results, the variation trends with the angle of attack are basically similar. Therefore, the applied CFD method with the SST model has been validated. Concerning the result provided by the $k-\varepsilon$ model, it shows good agreement at low angles of attack, presenting a behaviour similar to that of the SST model. However, it fails to give accurate predictions on both lift and drag coefficients, due to its failure on flow separation at higher angles of attack. Furthermore, in order to examine the effect from the computation domain, doubling the computation domain from $10 c$ to $20 c$ does not show any evident difference for $C_{L}$ or $C_{D}$, which can be observed by the comparison between them for some angles of attack.

\subsection{Control effect of SJ}

Figure 6 shows the time series of lift and drag coefficients of S809 aerofoil in five jet periods $(T)$ in three stall conditions: critical stall $\left(\alpha=11^{\circ}\right)$, deep stall $\left(\alpha=19^{\circ}\right)$ and complete stall $\left(\alpha=23^{\circ}\right)$ for both with and without SJ. Generally speaking, the aerodynamic forces of the aerofoil vary periodically with the blowing and sucking of the jet during the process, indicating that the control effect of SJ on flow separation is different at different times of blowing and sucking. When the aerofoil is in the critical stall condition (figure 6a), the lift coefficient increases and the drag coefficient decreases slightly after adding the jet. When the aerofoil is in the deep stall (figure 6b), both the increase in lift coefficient and the decrease in drag coefficient caused by the jet flow are larger than those under the critical stall condition, denoting more evident improvements in the 


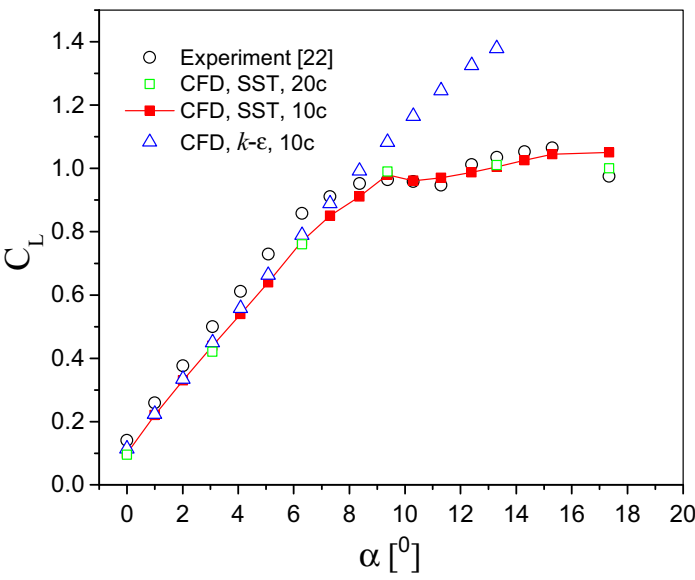

(a) Lift coefficient

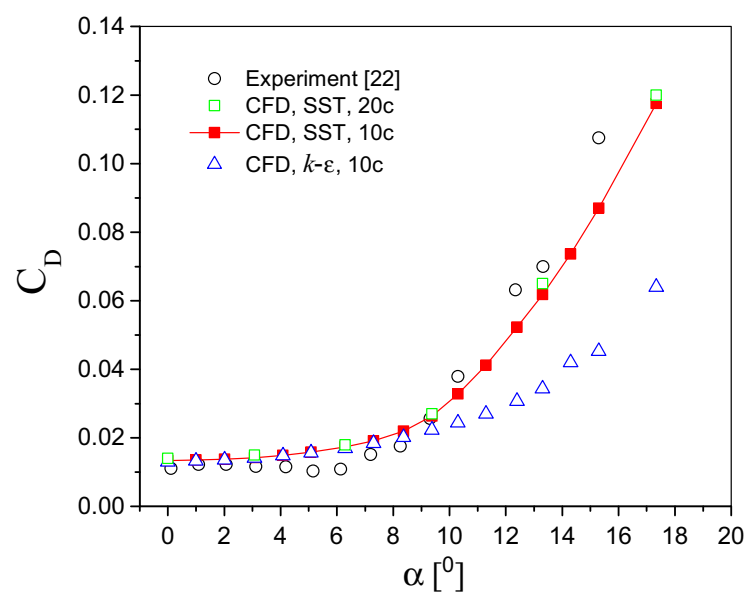

(b) Drag coefficient

Figure 5. Comparison between CFD and experimental results.

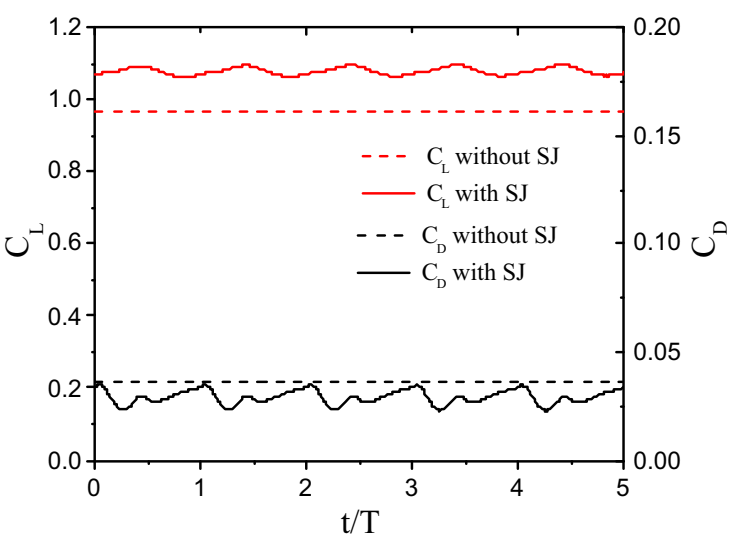

(a) $\alpha=11^{\circ}$

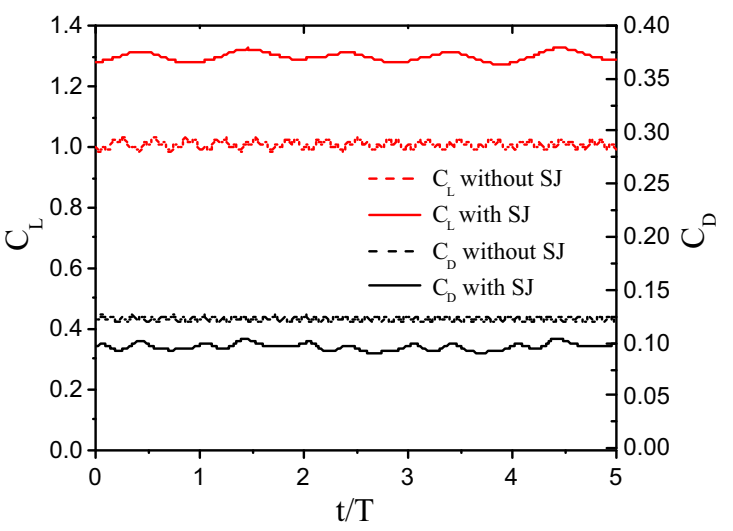

(b) $\alpha=19^{\circ}$

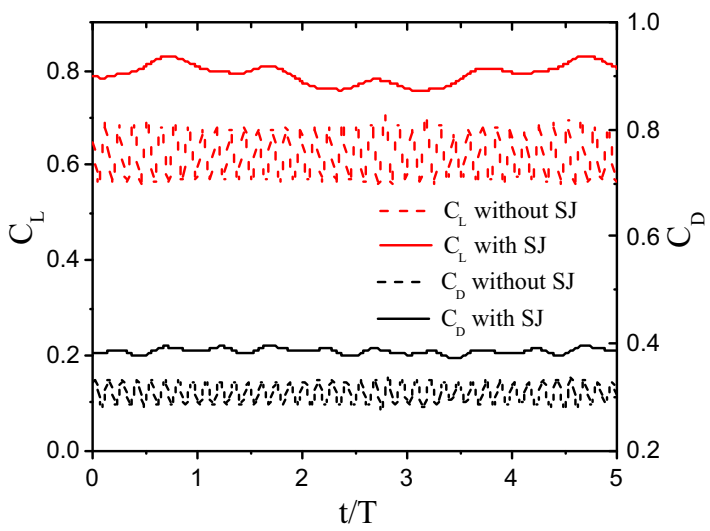

(c) $\alpha=23^{\circ}$

Figure 6. Comparison of lift and drag coefficients.

aerofoil performance. However, for the complete stall condition shown in figure $6 \mathrm{c}$, the effect of jet on aerofoil flow is very limited; the lift coefficient increases with the addition of jet, but the drag coefficient also increases. Therefore, the parameters of the jet need further optimization. 


\subsection{SJ optimization}

The OED method is a scientific method that combines mathematical statistics with orthogonality principle, arranges multi-factor experiments reasonably, reduces the number of experiments and shortens the period of experiments. It is based on mathematical statistics and probability theory, in which experiments are arranged according to certain rules. According to uniformity and orthogonality, the orthogonality table is employed to design the experiment scheme. The OED method has been proven to be an effective mathematical method for multi-factor experimental design [26]. Therefore, it has been applied to optimize the jet controlling parameters to achieve a better control on flow separation for the aerofoil under the deep stall condition $\left(\alpha=19^{\circ}\right)$. The frequency $(A)$, angle $(B)$ and maximum velocity $(C)$ of the $\mathrm{SJ}$ are selected to be the orthogonal design factors, and four levels are selected for each design factor, as shown in table 1.

Based on the factor levels in table 1, an orthogonal table L16 $\left(4^{3}\right)$ is chosen for the orthogonal experimental scheme, in order to examine the effect on flow control of the aerofoil for each factor. L16 $\left(4^{3}\right)$ denotes that three parameters and four levels for each parameter can be investigated within 16 experiments. The value of $C_{L} / C_{D}$ is selected to be the evaluation index to judge the flow control effect. Note that $C_{L} / C_{D}$ is dependent on time, the time-averaged value has therefore been used. The condition and corresponding result for each experiment are given in table 2. Four important parameters in a range analysis are listed: $K 1, K 2, K 3$ and $K 4$, which are defined as the average value of the evaluation index $C_{L} / C_{D}$ of four levels in each factor. In addition, another parameter $R$ is defined as the range between the maximum and minimum of $K_{i}(i=1,2,3$ and 4$)$ in the column for the corresponding factor. It indicates directly the effect: the larger, the greater effect on flow control. Therefore, it is obvious that the most important factor affecting jet efficiency is the jet velocity, followed by the jet angle and the jet frequency.

Figure 7 presents the effect of the three factors on jet efficiency. It is clear that the best jet scheme is A3B2C4, with the frequency of $75[\mathrm{~Hz}]$, the angle of $45^{\circ}$ and the maximum jet velocity of $3 U_{\infty}$ for this stall condition $\left(\alpha=19^{\circ}\right)$.

Table 1. Factor and level table for the jet.

\begin{tabular}{cccc}
\hline & \multicolumn{3}{c}{ Factor level } \\
\cline { 2 - 4 } Level & $A$ (frequency, Hz) & $B$ (angle) & $C$ (max. velocity) \\
\hline 1 & 25 & $30^{\circ}$ & $0.75 U_{\infty}$ \\
2 & 50 & $45^{\circ}$ & $1.5 U_{\infty}$ \\
3 & 75 & $60^{\circ}$ & $2.25 U_{\infty}$ \\
4 & 100 & $90^{\circ}$ & $3 U_{\infty}$ \\
\hline
\end{tabular}

Table 2. $\mathrm{L}_{16}$ orthogonal table for the jet.

\begin{tabular}{|c|c|c|c|c|}
\hline & \multicolumn{3}{|c|}{ Factor level } & \multirow{2}{*}{$\begin{array}{l}\text { Result } \\
C_{L} / C_{D}\end{array}$} \\
\hline & $A$ (frequency) & $B$ (angle) & $C$ (max. velocity) & \\
\hline 1 & 1 & 1 & 1 & 10.5 \\
\hline 2 & 1 & 2 & 2 & 9.9 \\
\hline 3 & 1 & 3 & 3 & 11.1 \\
\hline 4 & 1 & 4 & 4 & 10.7 \\
\hline 5 & 2 & 1 & 2 & 11.1 \\
\hline 6 & 2 & 2 & 1 & 10.4 \\
\hline 7 & 2 & 3 & 4 & 15.8 \\
\hline 8 & 2 & 4 & 3 & 10.3 \\
\hline 9 & 3 & 1 & 3 & 13.5 \\
\hline 10 & 3 & 2 & 4 & 16.9 \\
\hline 11 & 3 & 3 & 1 & 10.5 \\
\hline 12 & 3 & 4 & 2 & 10.2 \\
\hline 13 & 4 & 1 & 4 & 15.8 \\
\hline 14 & 4 & 2 & 3 & 14.1 \\
\hline 15 & 4 & 3 & 2 & 10.1 \\
\hline 16 & 4 & 4 & 1 & 10.2 \\
\hline$K 1$ & 10.6 & 12.7 & 10.4 & \\
\hline$K 2$ & 11.9 & 12.8 & 10.3 & \\
\hline$K 3$ & 12.8 & 11.9 & 12.3 & \\
\hline$K 4$ & 12.6 & 10.4 & 14.8 & \\
\hline$R$ & 2.2 & 2.4 & 4.5 & \\
\hline
\end{tabular}

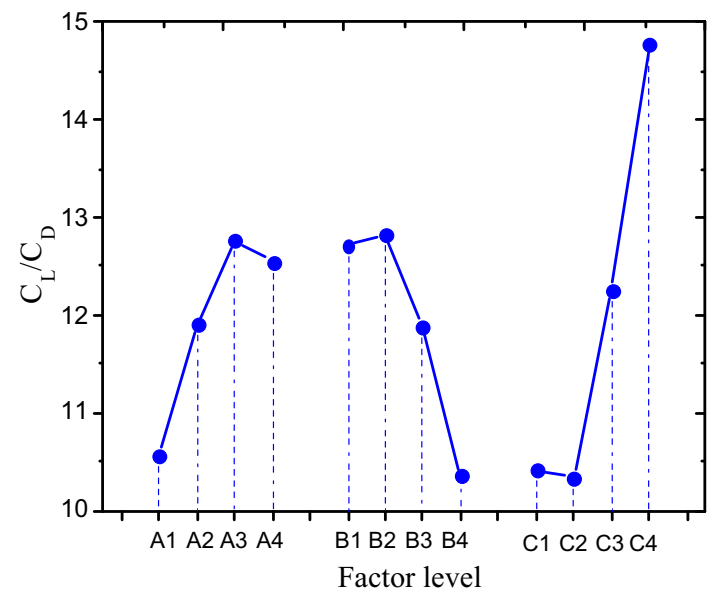

Figure 7. Relationship between factor level and jet efficiency.

Table 3. Comparison of lift-to-drag ratio.

\begin{tabular}{lccr}
\hline Case & $\alpha=11^{\circ}$ & $\alpha=19^{\circ}$ & $\alpha=23^{\circ}$ \\
\hline No control & 26.4 & 10.5 & 2.0 \\
With SJ & 37.1 & 13.5 & 2.1 \\
With optimized SJ & 48.3 & 16.9 & 12.6 \\
\hline
\end{tabular}


Table 3 shows the comparison of lift-to-drag ratio before and after jet optimization, and the value without SJ application is provided as well. The optimization on jet parameters has improved the aerofoil performance for all three stall conditions: the lift-to-drag ratio $C_{L} / C_{D}$ has been increased from 37.1 to 48.3 at $\alpha=11^{\circ}$, from 13.5 to 16.9 at $\alpha=19^{\circ}$. Especially for the complete stall condition at $\alpha=23^{\circ}$, the value of $C_{L} / C_{D}$ is slightly increased by applying the SJ without optimization, from 2.0 to 2.1 . However, this value has been enlarged to 12.6 after the jet optimization, denoting that the optimized jet scheme can improve further the aerodynamic performance of the aerofoil even in the complete stall condition.

Figure 8 compares the pressure coefficient $C_{P}$ on the aerofoil surface at different angles of attack, with the value being time-averaged during one period of the jet. For the cases with $\mathrm{SJ}$, an abrupt change in $C_{P}$ is found on the suction surface (upper surface) of the aerofoil, in the range
$12-13 \% c$ where the jet hole is located. It is observed that flow starts to separate from the aerofoil at about $55 \% \mathrm{c}$ for $\alpha=11^{\circ}, 40 \% c$ for $\alpha=19^{\circ}$ and nearly from leading edge for $\alpha=23^{\circ}$, judged by the zero slope of $C_{P}$ on the aerofoil upper surface and identified by checking local velocity vectors. However, this has been improved by applying the SJ, and further improvement has been obtained after the optimization on jet parameters. After optimization, the pressure difference between the upper and lower surfaces of the aerofoil has been increased, which is more pronounced for the complete stall condition at $\alpha=23^{\circ}$ shown in figure $8 \mathrm{c}$, resulting in a great increase in lift-to-drag ratio of the aerofoil.

The vorticity contours before and after optimization of SJ parameters are compared in figure 9 for the mentioned three stall conditions, and the one without application of SJ is given as well. It is obvious that the region with high magnitude of vorticity has been reduced by the application

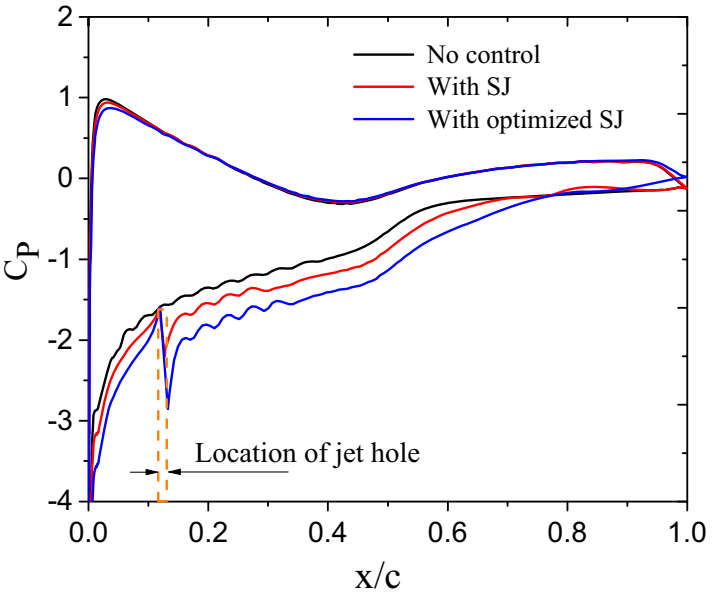

(a) $\alpha=11^{\circ}$

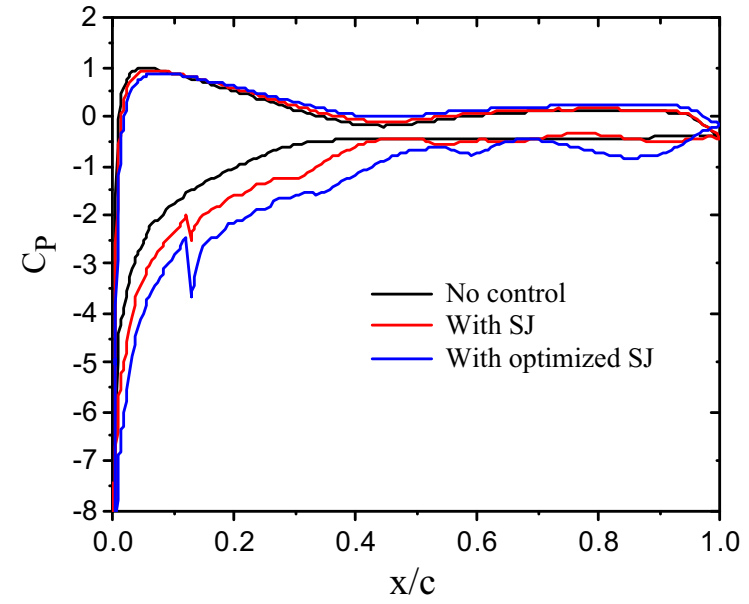

(b) $\alpha=19^{\circ}$

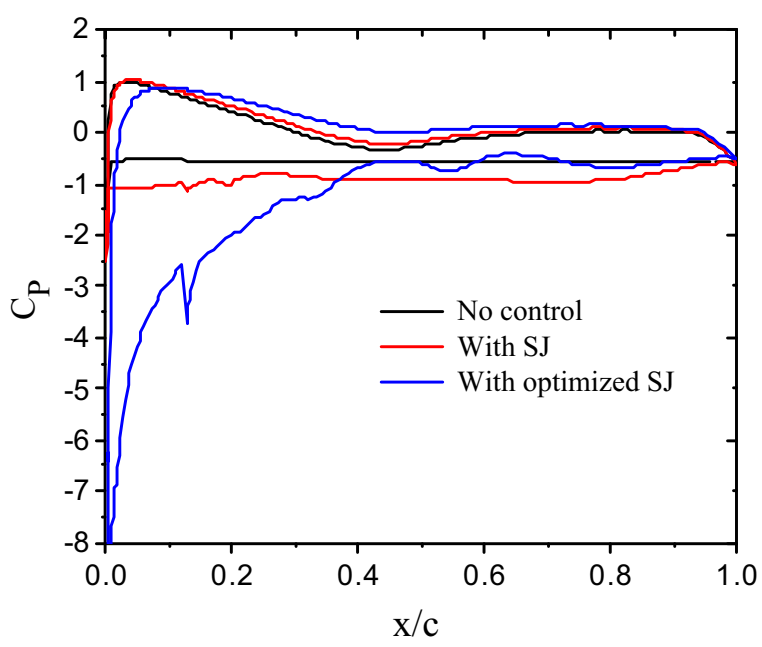

(c) $\alpha=23^{\circ}$

Figure 8. Comparison of time-averaged pressure coefficient before and after jet optimization at different angles of attack. 


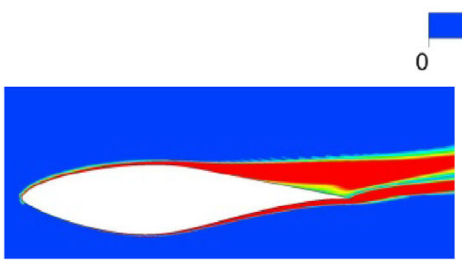

(a) $\alpha=11^{\circ}$, no SJ

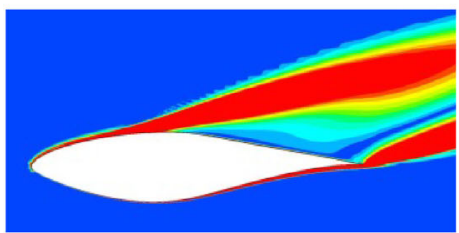

(d) $\alpha=19^{\circ}$, no SJ

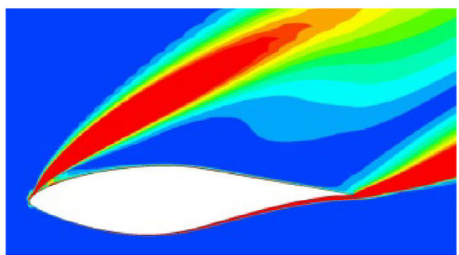

(g) $\alpha=23^{\circ}$, no SJ

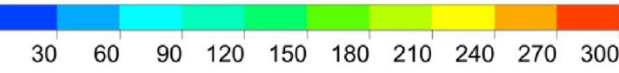

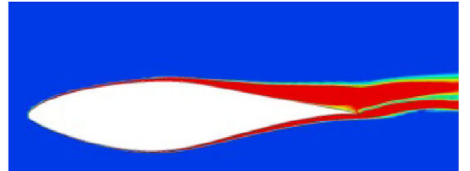

(b) $\alpha=11^{\circ}$, with SJ

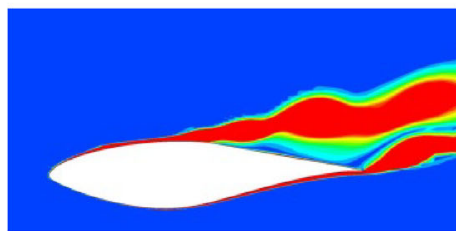

(e) $\alpha=19^{\circ}$, with SJ

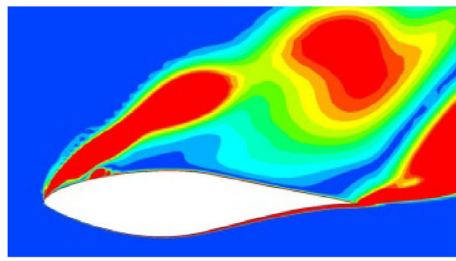

(h) $\alpha=23^{\circ}$, with SJ

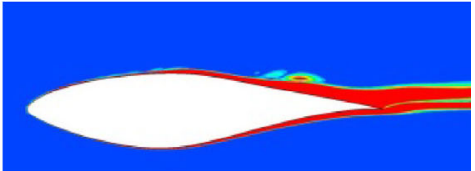

(c) $\alpha=11^{\circ}$, with optimized SJ

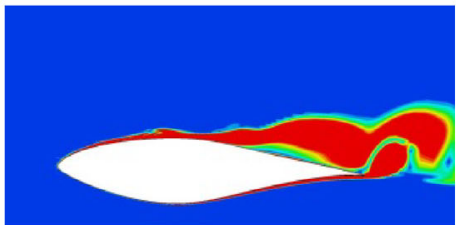

(f) $\alpha=19^{\circ}$, with optimized SJ

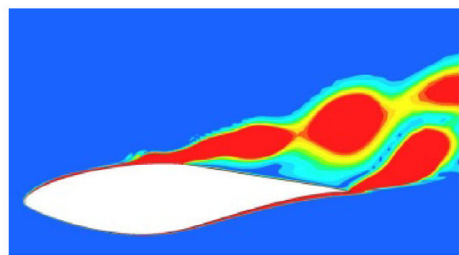

(i) $\alpha=23^{\circ}$, with optimized SJ

Figure 9. Vorticity contours at different stall conditions.

of SJ, further shrunk by the optimization of SJ. This trend becomes more evident with the increase of $\alpha$ from $11^{\circ}$ to $23^{\circ}$. At the deep stall condition $\left(\alpha=19^{\circ}\right)$, shown in figure $9 \mathrm{e}$ and $\mathrm{f}$, the jet vortex before jet optimization is formed directly at the jet hole, and detaches from the aerofoil upper surface at about $50 \%$ chord length. This results in a limited effect on controlling flow separation. After jet optimization, the vortex produced by the jet develops along the upper surface of the aerofoil and detaches from it near the trailing edge of the aerofoil. The jet vortex attaches completely the upper surface of the aerofoil during its development. The mixing of the low-velocity fluid in the boundary layer of the separation zone with the high-velocity fluid in the mainstream has delayed the flow separation and therefore increased the lift-to-drag ratio. This phenomenon become more evident for the complete stall condition $\left(\alpha=23^{\circ}\right)$ shown in figure $9 \mathrm{~h}$ and $\mathrm{i}$.

\subsection{Dual SJS}

Compared with one single jet, dual SJs can produce higher velocity and larger momentum, which is important for the flow control at high angles of attack. For the purpose of comparison, the effect of dual SJs on flow control for the aerofoil has been examined. The location of the first jet is kept unchanged, with an additional one located on the upper surface of the aerofoil, with a distance of $14 \% c$ to the aerofoil leading edge. The optimized jet parameters of the SJ are adopted: $f=75 \mathrm{~Hz}, V_{\max }=3 U_{\infty}$ and $\theta=45^{\circ}$.
Figure 10 compares streamlines under different schemes of SJ at different angles of attack, with contours coloured by pressure. At the angle of attack $\alpha=11^{\circ}$, flow separation occurs on the upper surface of the aerofoil for the case without SJ, starting from about $60 \% c$ and extending downstream to the trailing edge (figure 10a). Both single and dual jets have eliminated the flow separation, with similar pressure distribution on the aerofoil surface (figure $10 \mathrm{~b}$ and $\mathrm{c}$ ). At $\alpha=19^{\circ}$, with the application of SJ, the flow separation in deep stall has been greatly reduced, and the pressure on the aerofoil upper surface has been decreased (figure 10d-f). This produces a greater pressure difference between the aerofoil upper and lower surfaces, resulting in a bigger lift force, which is more pronounced for the dual jets. At the complete stall condition occurring at $\alpha=23^{\circ}$ (figure $10 \mathrm{~g}$ ), the application of dual jets is observed to have a much better effect on the improvement of flow separation (figure $10 \mathrm{~h}$ and $\mathrm{i}$ ); consequently a bigger lift-to-drag ratio $C_{L} / C_{D}$ can be expected, as shown in table 4 .

\section{Conclusions}

In this paper, the SJ technology has been employed for flow control at a high-Reynolds number of $\operatorname{Re}=1 \times 10^{6}$ for the S809 aerofoil, with the OED method being adopted for optimization. The main conclusions can be drawn as follows: 


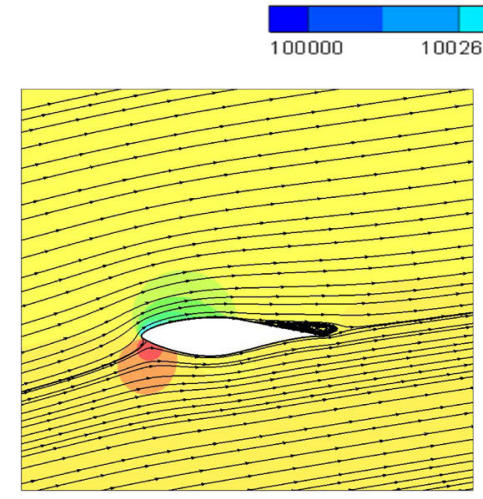

(a) $\alpha=11^{\circ}$, no synthetic jet

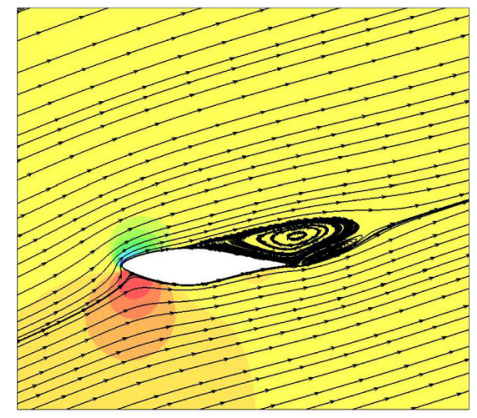

(d) $\alpha=19^{\circ}$, no synthetic jet

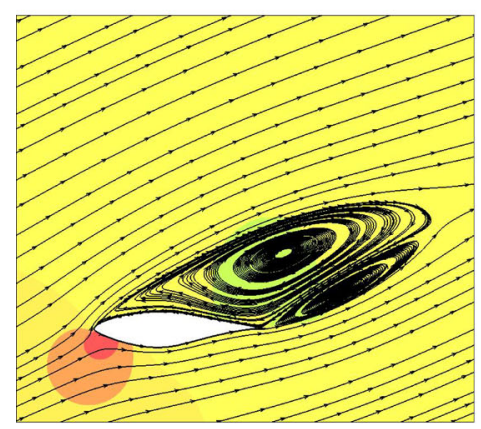

(g) $\alpha=23^{\circ}$, no synthetic jet

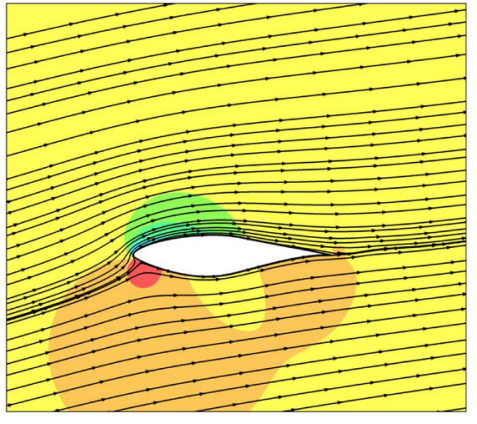

(b) $\alpha=11^{\circ}$, single jet

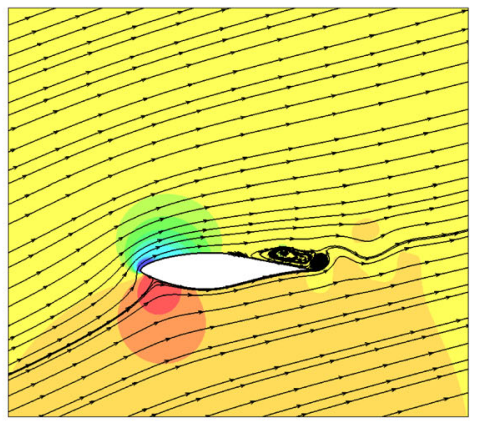

(e) $\alpha=19^{\circ}$, single jet

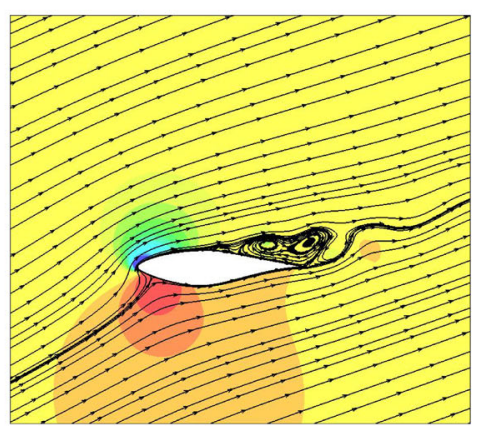

(h) $\alpha=23^{\circ}$, single jet

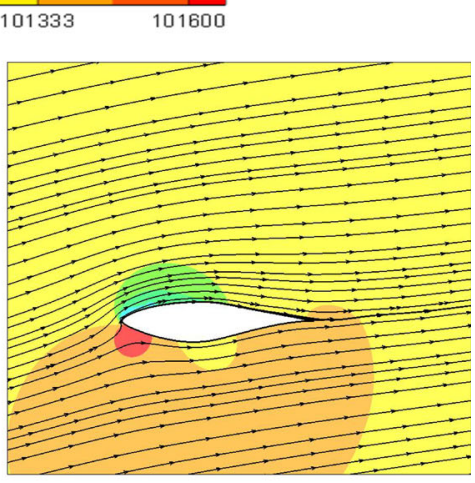

(c) $\alpha=11^{\circ}$, dual jets

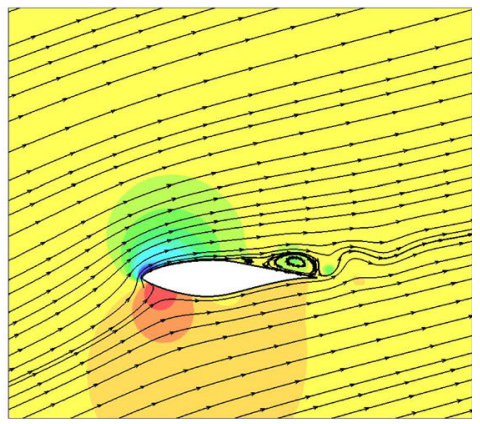

(f) $\alpha=19^{\circ}$, dual jets

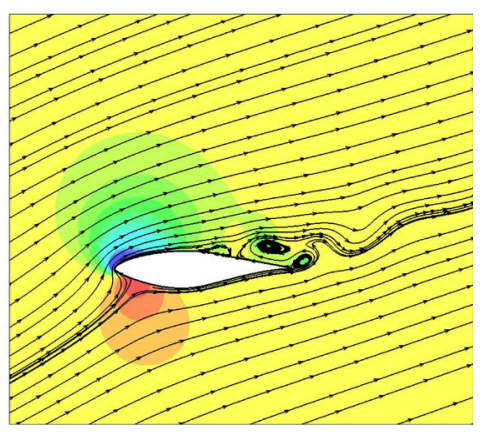

(i) $\alpha=23^{\circ}$, dual jets

Figure 10. Streamlines under different schemes of synthetic jet at different angles of attack; contours coloured by pressure.

Table 4. Comparison of lift-to-drag ratio for different jet schemes.

\begin{tabular}{lccr}
\hline Case & $\alpha=11^{\circ}$ & $\alpha=19^{\circ}$ & $\alpha=23^{\circ}$ \\
\hline No jet & 26.4 & 10.5 & 2.0 \\
Single jet & 48.3 & 16.9 & 12.6 \\
Dual jets & 69.2 & 19.4 & 14.4 \\
\hline
\end{tabular}

1. The aerodynamic performance of the aerofoil has been considerably improved by the introduction of SJ. The flow separation can be eliminated or reduced significantly by the jet, due to the mixing of low-energy fluid in boundary layer with high-energy fluid produced by the SJ, which impedes the formation of flow separation. The lift-to-drag ratio has been increased from 26.4 to 48.3 for the critical stall condition at $\alpha=11^{\circ}$, from 10.5 to 16.9 for the deep stall condition at $\alpha=19^{\circ}$ and from 2.0 to 12.6 for the complete stall condition at $\alpha=23^{\circ}$. Therefore, the critical angle of attack of the aerofoil has been considerably increased.

2. The maximum jet velocity of the SJ has the biggest effect on controlling flow separation, followed by the jet angle, then by the jet frequency. After optimization on jet parameters based on the OED method, further improvement on flow separation control has been achieved. 
3. Compared with single SJ, the dual jets can produce much better improvement on flow separation control of the aerofoil, especially at the complete stall condition. However, the configuration parameters of dual jets, such as the relative position between them, can be further optimized.

\section{Acknowledgements}

This research was supported by the National Key R\&D Program of China (2018YFB1501900), the National Natural Science Foundation of China (Grant Nos. 51679195 and 51339005) and Natural Science Basic Research Plan in Shaanxi Province of China (Program No. 2018JM5102).

\section{Nomenclature}

$V_{\text {jet }} \quad$ jet velocity

$\alpha \quad$ angle of attack

$V_{\max } \quad$ maximum jet velocity

$c \quad$ chord length of aerofoil

$C_{P} \quad$ pressure coefficient

$U_{\infty} \quad$ incoming flow velocity

$h \quad$ jet hole width

$C_{D} \quad$ drag coefficient

$C_{L} \quad$ lift coefficient

$f \quad$ frequency of synthetic jet

$F_{L} \quad$ lift force

$F_{D} \quad$ drag force

$P \quad$ pressure

$P_{o} \quad$ standard atmospheric pressure

$\operatorname{Re} \quad$ Reynolds number, $\operatorname{Re}=\rho c U_{\infty} / \mu$

$\mu \quad$ dynamic viscosity

$\rho \quad$ density

$\theta \quad$ jet angle

$x \quad$ distance on chord starting from the leading edge

SJ synthetic jet

$t \quad$ time

$T \quad$ synthetic jet period

\section{References}

[1] Amitay M, Smith D R, Kibens V, David E P and Ari G 2001 Aerodynamic flow control over an unconventional airfoil using synthetic jet actuators. AIAA Journal 39(3): 361-370

[2] Tuck A and Soria J 2004 Active flow control over a NACA0015 airfoil using a ZNMF jet. In: Proceedings of the 15th Australasian Fluid Mechanics Conference, Sydney, Australia, pp. 1-4

[3] You D and Moin P 2008 Active control of flow separation over an airfoil using synthetic jets. Journal of Fluids and Structures 24: 1349-1357

[4] Wu J Z, Lu X Y, Denny A G, Fan M and Wu J 1998 Poststall flow control on an airfoil by local unsteady forcing. Journal of Fluid Mechanics 31: 21-58
[5] Duvigneau R and Visonneau M 2006 Optimization of a synthetic jet used for aerodynamic stall control. Computers and Fluids 35(6): 624-638

[6] Zdenek T and Vaclav T 2003 Annular synthetic jet used for impinging flow mass transfer. International Journal of Heat and Mass Transfer 46(17): 3291-3297

[7] Guo D H, Cary A W and Agarwal R K 2003 Numerical simulation of vectoring of a primary jet with a synthetic jet. AIAA Journal 41(12): 2364-2370

[8] Washburn A E and Amitay M 2004 Active flow control on the stingray UAV: physical mechanisms. AIAA 2004-0745

[9] Shaw L, Smith B and Saddoughi S 2006 Full scale flight demonstration of active flow control of a pod wake. AIAA 2006-3183

[10] Smith B L and Glezer A 1998 The formation and evolution of synthetic jets. Physics of Fluids 10(9): 2281-2297

[11] Cannelle F and Amity M 2005 Synthetic jets: spatial evolution and transitory behavior. AIAA-2005-102

[12] Vargas Y, Finley T, Mohseni K, et al 2006 Flow characterization of a synthetic jet. AIAA-2006-1442

[13] Guo F and Zhong S 2006 PIV investigation of the characteristics of micro-scale synthetic jets. AIAA-2006-3183

[14] Shuster J M and Simth D R 2007 Experimental study of the formation and scaling of a round synthetic jet. Physics of fluids 19(4):1-21

[15] Seifert A, Tunia L and Pack G 1999 Oscillatory excitation of unsteady compressible flows over airfoils at flight Reynolds numbers. AIAA 99-0925

[16] Qin Y, Song Y P, Wang R Y, et al 2017 Flow separation control on a highly loaded compressor cascade using endwall synthetic jets. Journal of Aerospace Engineering 232(11): 2059-2075

[17] Kamari D and Tadjfar M 2018 Synthetic jet flow control optimization on SD7003 airfoil at low Reynolds number. In: Proceedings of the ASME 2018 Fluids Engineering Division Summer Meeting, Montreal, Canada, pp. 1-8

[18] Velasco D, Mejia O L and Lain S 2017 Numerical simulations of active flow control with synthetic jets in a Darrieus turbine. Renewable Energy 113: 129-140

[19] Gilarranz J L and Rediniotis O K 2001 Compact, high-power synthetic jet actuators for flow separation control. AIAA Paper 2001-0737

[20] Gilarranz J L, Traub L W and Rediniotis O K 2005 A new class of synthetic jet actuators, part II: application to flow separation control. Journal of Fluids Engineering 127: 377-387

[21] Zhao Q J, Ma Y Y and Zhao G Q 2017 Parametric analyses on dynamic stall control of rotor airfoil via synthetic jet. Chinese Journal of Aeronautics 30(6): 1818-1834

[22] Somers D 1997 Design and experimental results for the S809 airfoil NREL/SR-440-6918. Tech. Rep. NREL

[23] Lan A Q, Wang Z H, Yu H S and Xi G 2016 Numerical investigation for influence of synthetic jet actuation location on airfoil flow separation control at large attack angle. Journal of Xi'an Jiaotong University 50(7): 110-117

[24] Zhang P F and Wang J J 2008 Numerical simulation on flow control of stalled NACA0015 airfoil with synthetic jet actuator in recirculation region. Journal of Beijing 
University of Aeronautics and Astronautics 34(4): 443-446

[25] Menter F R 1994 Two-equation eddy-viscosity turbulence models for engineering applications. AIAA-Journal 32(8): 1598-1605
[26] Deng L N, Feng B and Zhang Y 2018 An optimization method for multi-objective and multi-factor designing of a ceramic slurry: combining orthogonal experimental design with artificial neural networks. Ceramics International 44: 15918-15923 